\title{
Pulmonary Alveolar Proteinosis
}

National Cancer Institute

\section{Source}

National Cancer Institute. Pulmonary Alveolar Proteinosis. NCI Thesaurus. Code C85037.

A rare lung disorder characterized by the filling of the pulmonary alveoli with

proteinaceous material which stains positive with periodic acid-Schiff stain. It may be idiopathic or secondary due to hematologic malignancies or the inhalation of mineral dusts. Signs and symptoms include dyspnea, cough and low grade fever. 